\title{
Estudio del mecanismo de eliminación de magnesio de aleaciones Al-Si en estado líquido mediante inyección de minerales base sílice $^{(\bullet)}$
}

\author{
R. Muñoz-Arroyo*, J.C. Escobedo-Bocardo*, H.M. Hernández-García*, D.A. Cortés-Hernández*, \\ M. Terrones-Maldonado**, A. Rodríguez-Pulido** y J.L. Hernández-Piñero***
}

\begin{abstract}
Resumen Se empleó el método de inyección sumergida de polvos por medio de un gas de arrastre inerte (Ar) con el fin de eliminar el magnesio de la aleación Al-Si A380 a $750^{\circ} \mathrm{C}$. Los polvos inyectados al baño de metal fundido fueron zeolita mineral, arena sílice y mezclas de ambas. Las variables de respuesta medidas fueron el contenido de magnesio en el baño metálico respecto al tiempo de inyección y las mermas de metal al final de cada experimento. En el análisis de resultados, la mezcla sílice:zeolita 66:34 \% e.p. obtuvo la mayor eficiencia, lográndose una disminución en el contenido de magnesio en el baño metálico de 1 a 0.0066 \% e.p. Los productos de reacción se analizaron por difracción de rayos-X, microscopía electrónica de barrido y de transmisión. Los resultados de estos análisis y el empleo del paquete termodinámico FactSage, versión 6, permitieron justificar el mecanismo de reacción entre los minerales y el aluminio líquido.
\end{abstract}

Palabras clave Zeolita; Sílice; Aluminio; Magnesio; Minerales.

\section{Study of the removal mechanism of magnesium from Al-Si liquid alloys using silica base minerals injection}

\begin{abstract}
In order to eliminate magnesium from an $\mathrm{A} 380 \mathrm{Al}-\mathrm{Si}$ alloy at $750^{\circ} \mathrm{C}$, the submerged powder injection method, using an inert carrier gas (Ar), was applied. The injected powders in the liquid aluminum bath were zeolite, silica and mixtures of zeolite-silica minerals. For each experiment the response variables were: eliminated magnesium versus injection time and quantity of drosses produced. Chemical analysis by atomic absorption spectrometry showed that mixtures of silica-zeolite $66: 34 \mathrm{wt} \%$ have the best results with regarding to the removal magnesium from 1 to 0.0066 wt $\%$. During the elimination of magnesium complex stoichiometry compounds were formed due to the reactions among zeolite, water steam and liquid aluminum. These compounds were analyzed by XRD, SEM and TEM. The results obtained, along with using the FactSage 6 thermodynamic software, allowed to elucidate the reaction mechanism between the minerals used and liquid aluminum.
\end{abstract}

Keywords

Zeolite; Silica; Aluminum; Magnesium; Minerals.

\section{INTRODUCCIÓN}

Se emplean diversos métodos para la eliminación de magnesio de la aleación Al-Si A380 de uso automotriz (donde se requiere un contenido máximo de 0,1 \% e.p. de magnesio). Los métodos más comunes son la cloración, el método electroquímico y la incorporación de polvos reactivos tales como algunos fluoruros $^{[1-4]}$. Sin embargo, estos métodos presentan algunas desventajas. El primero y el tercero de los métodos mencionados generan productos contaminantes al medio ambiente y al hombre. Mientras el segundo de ellos, aunque no produce este tipo de productos, resulta inviable debido a los altos costos de la energía eléctrica. Con base en lo anterior, la industria del aluminio secundario se ha estado enfocando al desarrollo de procesos que superen las limitaciones de los anteriores. Una opción la representa

\footnotetext{
(•) Trabajo recibido el día 22 de septiembre de 2009 y aceptado en su forma final del día 8 de agosto de 2010

* Centro de Investigación y de Estudios Avanzados del IPN, Unidad Saltillo, México.

${ }^{* *}$ Instituto Potosino de Investigación en Ciencia y Tecnología, San Luis Potosí, México.

*** Universidad Autónoma de Nuevo León, Nuevo León, México.
} 
el uso de polvos a base de sílice beneficiada, debido a que los vapores y productos generados en la operación de eliminación de magnesio no son contaminantes. Informes científicos, en la literatura, establecen el efecto del $\mathrm{SiO}_{2}$ en la remoción de magnesio ${ }^{[5-7]}$. La eliminación del magnesio se lleva a cabo considerando la siguiente reacción $(1)^{[8]}$ :

$$
\begin{aligned}
& \mathrm{Mg}_{(l)}+2 \mathrm{Al} \mathrm{I}_{(\mathrm{l})}+2 \mathrm{SiO}_{2(\mathrm{~s})} \rightarrow \mathrm{MgAl}_{2} \mathrm{O}_{4(\text { espinela })}+2 \mathrm{Si}_{(\mathrm{s})} \\
& \Delta \mathrm{G}^{\circ}{ }_{750^{\circ} \mathrm{C}}=-377.09 \mathrm{~kJ}
\end{aligned}
$$

No obstante, la sílice requiere de procesos de preparación para beneficiarla, lo que deviene en un costo adicional además de que la cinética de remoción de magnesio utilizando sílice es relativamente lenta.

Estudios recientes han demostrado la factibilidad de eliminar magnesio de aleaciones de aluminio líquidas inyectando zeolita y arena sílice, ambos minerales ricos en $\mathrm{SiO}_{2}$ (con un contenido mayor al 50 \% e.p. ${ }^{[5 \text { y } 8]}$. En la zona norte de México se localizan menas zeolíticas y de arena sílice, por lo que la abundancia de estos materiales hace viable su uso para eliminar magnesio de este tipo de aleaciones además de disminuir costos de tratamiento.

\section{DESARROLLO EXPERIMENTAL}

\subsection{Materiales empleados y procesamiento de minerales}

La composición química de los minerales de zeolita y sílice empleados se muestra en la tabla I. Los minerales fueron molidos en un molino de bolas y cribados a -150 mallas ASTM. Posteriormente, se usó un molino planetario con tarros de ágata para disminuir el tamaño de partícula a $150 \mu \mathrm{m}$. La composición química de la aleación de aluminio empleada para los experimentos fue: $\mathrm{Al}, 11,64$ \% e.p., Si, 0, 338 \%, e.p., Fe, 2,05 \% e.p., $\mathrm{Cu}, 1,00$ \% e.p., $\mathrm{Mg} 1,00 \%$ e.p y Ni, 0,54 \% e.p. Se seleccionó el argón de ultra-alta pureza (99.9999\%) para ser empleado como gas de arrastre en las pruebas de inyección.

\subsection{Inyección de minerales}

Para la fusión y el tratamiento de la aleación líquida, se empleó un horno eléctrico de inducción con un crisol de carburo de silicio de una capacidad para 12 $\mathrm{kg}$ de aluminio, equipado con controlador de temperatura. Para la inyección de polvos se utilizó un dispensador que controla la cantidad de polvo y gas a inyectar. Para el control de la inyección de los polvos y gas se utilizó una lanza de grafito de diámetro interno de 0,698 cm, posicionando la punta de la misma al $85 \%$ de la profundidad total del crisol. Durante los experimentos se mantuvieron constantes la carga metálica al horno (8 $\mathrm{kg}$ de aleación), la temperatura del baño metálico $\left(750^{\circ} \mathrm{C}\right)$, los flujos de gas y polvo alimentados $(12,84 \mathrm{~g} / \mathrm{min}$ de mineral por $4 \mathrm{l} / \mathrm{min}$ de argón) y el tamaño de partícula de los polvos (tamaño menor a150 $\mu \mathrm{m}$ ). Durante cada fusión, se extrajeron muestras del baño líquido cada $10 \mathrm{~min}$ hasta los $80 \mathrm{~min}$ de tratamiento. Al finalizar cada experimento, se extrajo una muestra de escoria de la superficie del baño líquido. Las escorias se analizaron por difracción de rayos$\mathrm{X}$ y las muestras metálicas por espectrometría de emisión atómica por chispa.

\subsection{Estudio de las reacciones en los minerales}

Para la realización de este estudio, se sumergieron en el baño metálico, a la temperatura de $750^{\circ} \mathrm{C}, 8$ cubos de zeolita de $3 \mathrm{~cm}$ por lado, con ayuda de una canastilla de acero recubierta con material refractario. Durante el proceso, se inyectó argón al baño metálico, para agitarlo. Se extrajo un cubo cada $10 \mathrm{~min}$ hasta completar $80 \mathrm{~min}$. Posteriormente, cada cubo se observó bajo el estereoscopio y, con la ayuda de un escalpelo, se le retiró la capa de reacción. Las

Tabla I. Análisis químico cuantitativo de los minerales base sílice

Table I. Quantitative chemical analysis of the silica base minerals used

\begin{tabular}{cccccccccc}
\hline Mineral & $\mathbf{K}$ & $\mathbf{C a}$ & $\mathbf{N a}$ & $\mathbf{S r}$ & $\mathbf{B a}$ & $\mathbf{A l}$ & $\mathbf{S i O}_{2}$ & $\mathbf{F e}$ & $\mathbf{M g}$ \\
\hline Zeolita & 1,41 & 1,43 & 0,058 & $<0,005$ & $<0,005$ & 6,080 & 56,090 & 1,510 & 0,400 \\
Sílice & 0,004 & 0,059 & 0,022 & $<0,200$ & $<0,200$ & 0,155 & 99,190 & 0,080 & 0,010 \\
\hline
\end{tabular}

* El porcentaje de $\mathrm{H}_{2} \mathrm{O}$ en la Zeolita es de $12,0180 \%$ e.p. 
capas de reacción se analizaron por difracción de rayos-X. Finalmente, con los productos de reacción encontrados y el empleo del paquete termodinámico FactSage versión 6, se procedió a establecer las condiciones termodinámicas de los mecanismos de reacción en eliminación de magnesio.

\subsection{Caracterización de muestras metálicas por microscopía electrónica de transmisión}

Se seleccionaron muestras metálicas para ser analizadas por microscopía electrónica de transmisión. Las muestras a analizar se prepararon en tres etapas. La primera constó de corte, desbaste y limpieza por ultrasonido hasta obtener muestras con un espesor de $150 \mu \mathrm{m}$. Se procedió, enseguida, al corte por ultrasonido de las muestras a la medida estándar para ser observadas al MET y, la última etapa, consistió en un ataque electrolítico usando una mezcla de 0,9 1 de etanol y 0,009 1 de ácido clorhídrico como reactivos de ataque químico, ésto, con el fin de revelar microestructura y fases presentes en el material. El ataque electroquímico se llevó a cabo con corrientes de 436 a $362 \mu \mathrm{A}$, a $50 \mathrm{~V}$, con tiempos de 28 a 56 s de inmersión en el electrolito. Finalmente, se analizaron las muestras por microscopía electrónica de transmisión.

\section{ANÁLISIS Y DISCUSIÓN DE RESULTADOS}

\subsection{Difracción de rayos $X$ de los minerales empleados}

La figura 1 muestra los resultados de los análisis por difracción de rayos-X de la zeolita y la sílice utilizadas. En la zeolita, se identificaron dos compuestos: $\mathrm{Ca}\left(\mathrm{Si}_{7} \mathrm{Al}_{2}\right) \mathrm{O}_{18} \cdot 6 \mathrm{H}_{2} \mathrm{O}$ (heulandita), que es clasificada co-

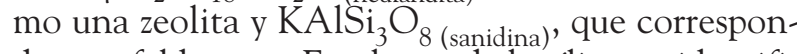
de a un feldespato. En el caso de la sílice, se identificó únicamente $\mathrm{SiO}_{2}$ (cuarzo) .

\subsection{Eliminación de magnesio empleando zeolita, sílice y mezclas de zeolita + sílice}

Las pruebas de eliminación de magnesio se realizaron con las condiciones especificadas anteriormente, empleando zeolita, sílice y mezclas sílice:zeolita 50:50 y 34:66 \%e.p. como agentes eliminadores de magnesio.

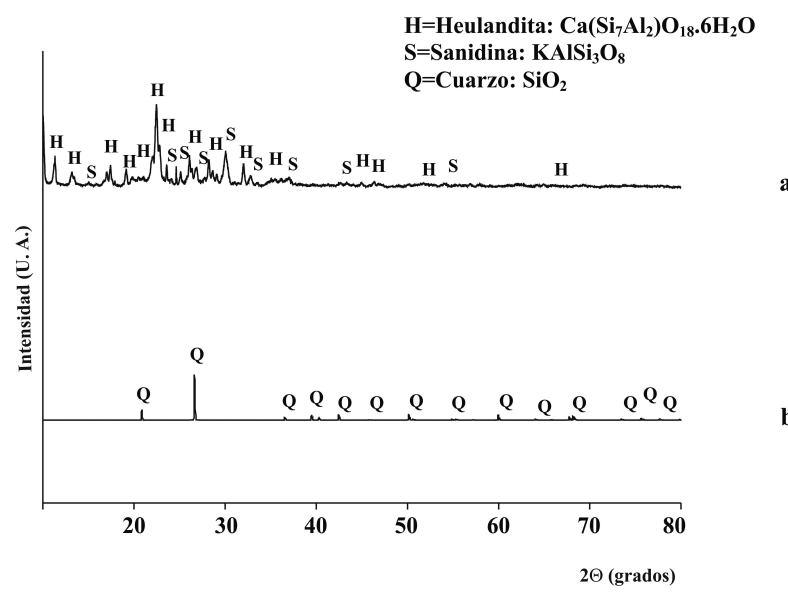

Figura 1. Difractogramas de los minerales empleados: a) zeolita y b) sílice.

Figure 1. X-ray diffraction patterns of the minerals used: a) zeolite and b) silica.

En la figura 2, se presenta la variación del contenido de magnesio en la aleación de aluminio con respecto al tiempo de inyección para las diferentes pruebas efectuadas, así como los valores calculados para la constante de velocidad de reacción $(\mathrm{k})$.

Se aprecia que los mejores resultados se obtuvieron con el empleo de la mezcla sílice:zeolita 34:66, con la zeolita pura y la mezcla sílice:zeolita 50:50, en ese orden. Se observó, además, que mediante el empleo de las mezclas de sílice:zeolita se generó una

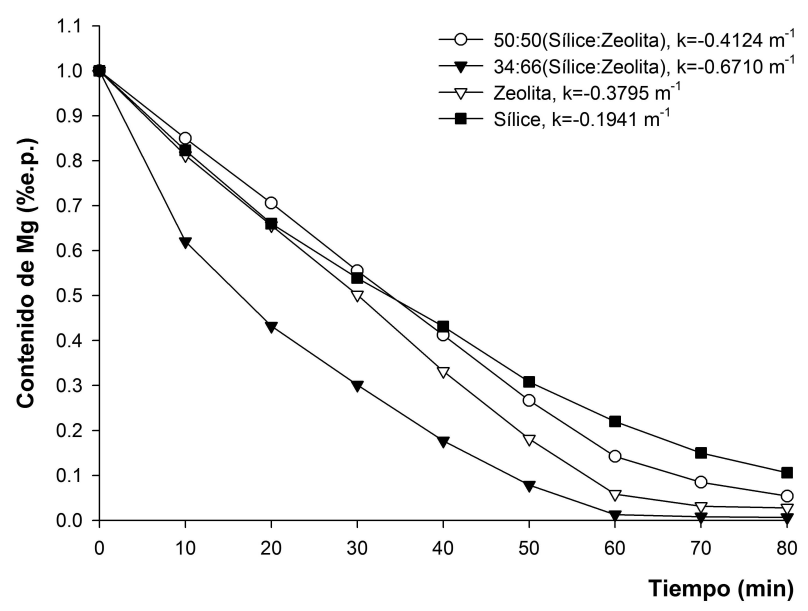

Figura 2. Variación del contenido de magnesio en el baño de aleación de aluminio en función del tiempo de inyección.

Figure 2. Injection experiments. magnesium content vs. injection time for the different solids tested. 
menor cantidad de escoria, formándose óxidos de alta estabilidad como son $\mathrm{MgAl}_{2} \mathrm{O}_{4 \text { (espinela) }}, \mathrm{MgO}_{\text {(peri- }}$

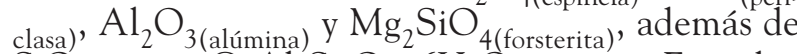
$\mathrm{SiO}_{2 \text { (cuarzo) }}$ y $\mathrm{CaAl} \mathrm{Si}_{7} \mathrm{O}_{18} .6 \mathrm{H}_{2} \mathrm{O}_{\text {(heulandita). }}$. Este último compuesto pertenece al grupo de la zeolita al igual que la $\mathrm{Ca}_{8.16} \mathrm{Si}_{36} \mathrm{O}_{72}\left(\mathrm{H}_{2} \mathrm{O}\right)_{21.8 \text { (clinoptilolita) y la }}$ $\mathrm{CaAlSi}_{2} \mathrm{O}_{8}$ (anortita).

Durante la eliminación de magnesio usando mezclas de sílice:zeolita se presentan dos efectos:

Primero, el efecto de la zeolita. La zeolita en su estado natural es un compuesto hidratado (el porcentaje de agua en la zeolita empleada es del 12 $\%$ e.p.). Por efecto de la temperatura, el agua en la zeolita se calienta y evapora fragmentado la partícula por expansión del vapor a través de los canales (Fig. 3). Además, el agua es un agente fuertemente oxidante que coadyuva a la eliminación del magnesio ${ }^{[8]}$.

Las fuerzas convectivas del horno, el flujo del gas de arrastre y la topografía de las maclas de Carlsbad en la zeolita, contribuyen a la conminución de las partículas, obteniéndose, incluso, tamaños menores a $80 \mu \mathrm{m}$ (Fig. 4 a) y b)). Las maclas de Carlsbad (Fig. 4 a)) presentan hábito tabular paralelo al plano (010); esta familia de planos presentan fragilidad en los materiales ${ }^{[9]}$. En la figura 4 a) se observa una partícula de zeolita antes de la inyección, con un tamaño aproximado de $165 \mu \mathrm{m}$. En la figura $4 \mathrm{~b}$ ) se observa una partícula de zeolita semireaccionada, obtenida a los 10 min de iniciar la inyección, con un tamaño aproximado de $50 \mu \mathrm{m}$.

El segundo efecto es el de la sílice. $\mathrm{El} \mathrm{SiO}_{2}$ reacciona con el magnesio disuelto en el baño líquido formándose productos de reacción que envuelven la partícula de sílice. En la figura 5 a) se observa una imagen, obtenida por electrones retrodispersados, de una partícula de sílice antes de ser inyectada, con un tamaño aproximado de 200 $\mu \mathrm{m}$. En la figura $5 \mathrm{~b}$ ) se observan partículas de sílice semi-reaccionadas, con tamaños entre 50 y $300 \mu \mathrm{m}$, obtenidas a los $80 \mathrm{~min}$ de iniciar la

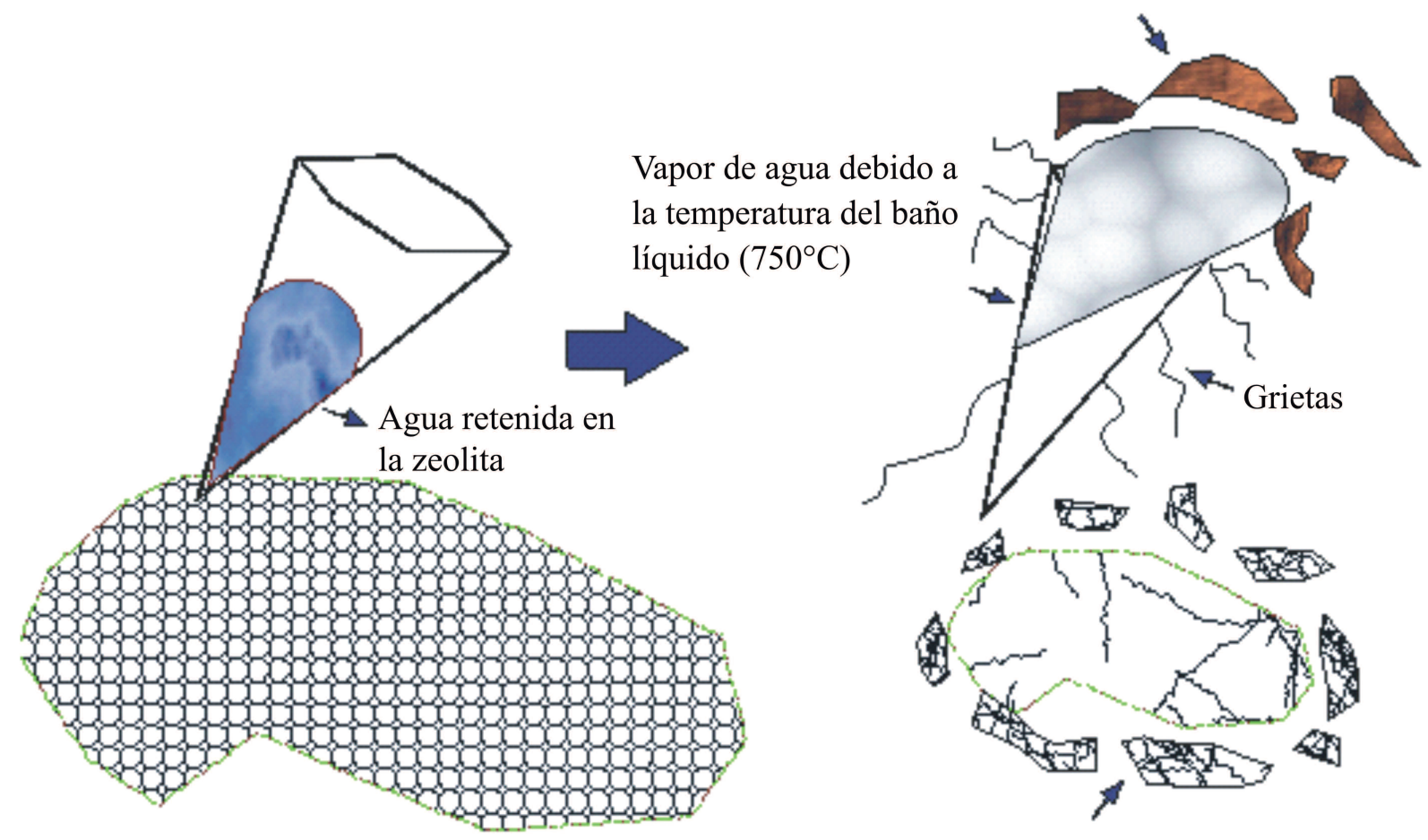

Fragmentos de zeolita y capas oxidadas sobre el mineral

Agrietamiento y fragmentación del mineral debido al efecto mecánico de la salida de vapor a través de los túneles de la zeolita

Figura 3. Efecto mecánico de expansión de la salida de agua en forma de vapor ${ }^{[8]}$.

Figure 3. Mechanical effect of the expansion of output of water steam ${ }^{[8]}$. 

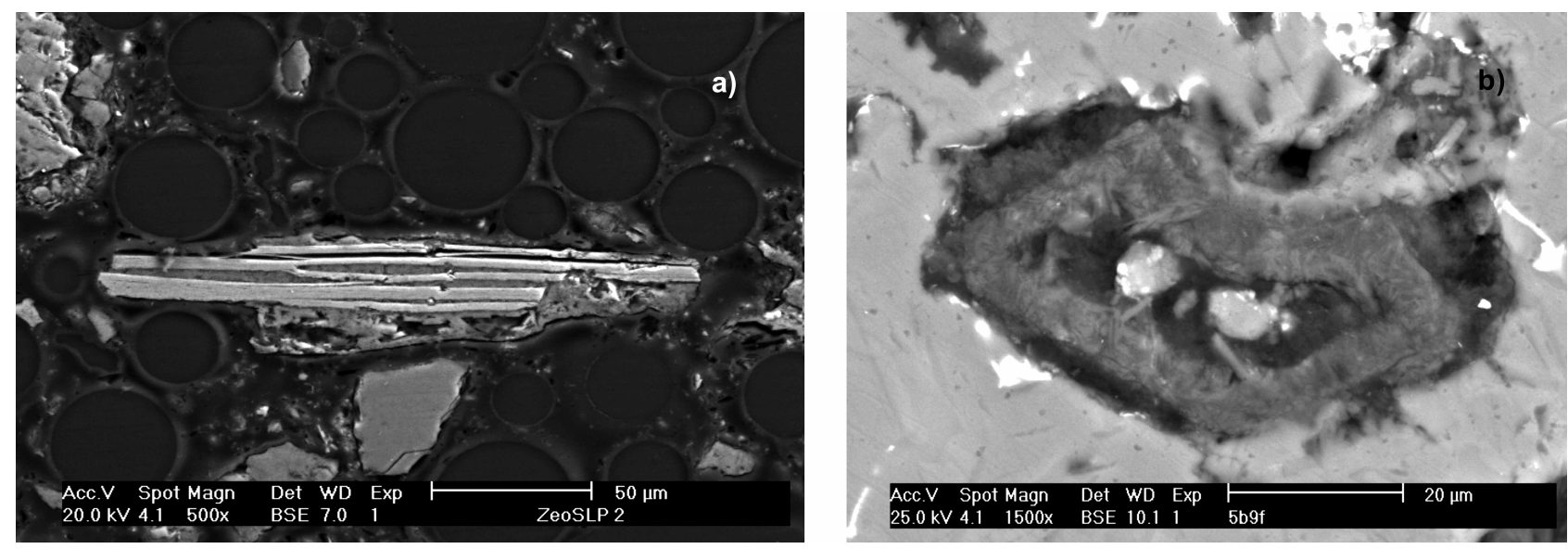

Figura 4. a) Partícula antes de la inyección de zeolita con maclas de Carlsbad; b) Partícula semireaccionada de zeolita a los 10 min de iniciar la inyección.

Figure 4. a) Initial zeolite particle shows topography of Carlsbad twins; b) Particle of semi-reacted zeolite after 10 min of starting the injection.
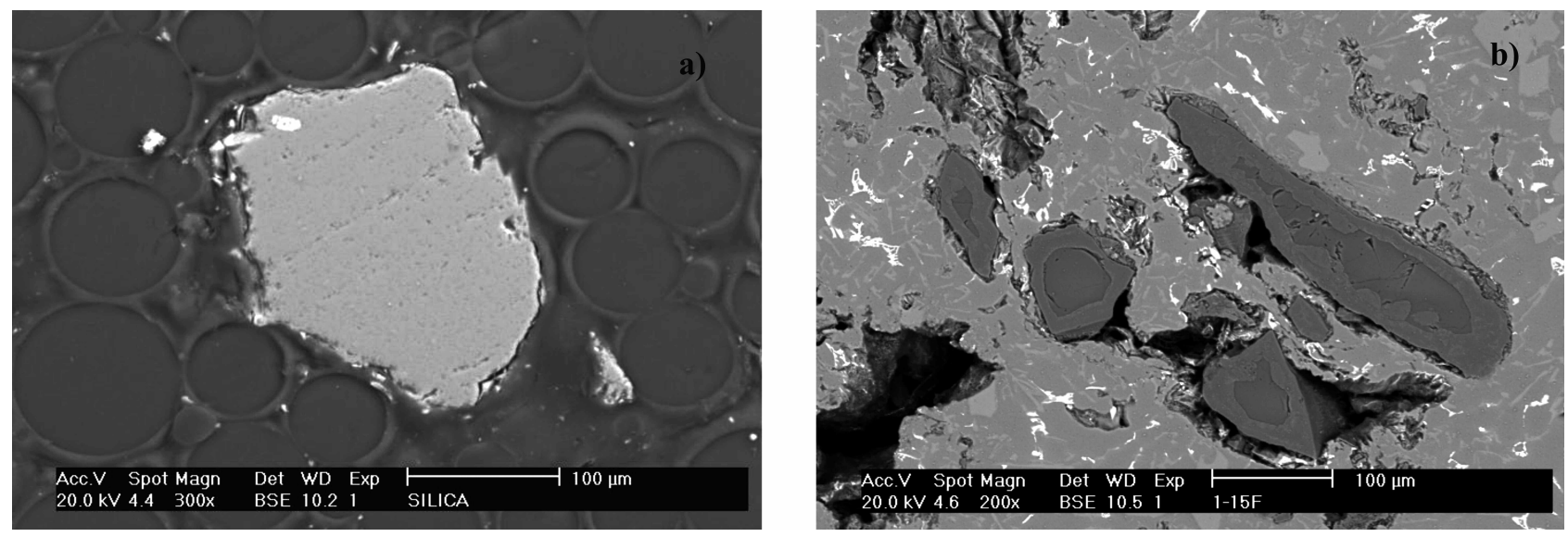

Figura 5. a) Partícula de sílice antes de ser inyectada; b) Partícula semireaccionada de sílice a los 80 min de iniciar la inyección.

Figure 5. a) Initial silica particle; b) Particle of semi-reacted silica after 80 min of starting the injection.

inyección. Es posible observar una capa de productos de reacción. La formación de esta capa de productos alrededor de la sílice explica el por qué de la relativamente baja velocidad de eliminación de magnesio cuando se emplea sílice.

En base a los procesos industriales que requieren una mayor eficiencia de eliminación de magnesio y la menor formación de escoria (máximo, del 10 \%), las mayores eficiencias se obtuvieron para la zeolita sola y para la mezcla 34, sílice:66, zeolita. Comparativamente, se aprecia que la mezcla es más viable de usar en un proceso industrial de fundición debido a la menor merma con respecto a la zeolita, de acuerdo a la tabla II.

\subsection{Planteamiento de reacciones químicas involucradas en la descomposición de la zeolita, calculadas mediante el software FactSage}

En la tabla III se reflejan los compuestos encontrados en la superficie de los cubos de zeolita, después de que éstos estuvieron sumergidos en el baño metálico por diferentes periodos de tiempo.

De acuerdo a los productos de reacción identificados por difracción de rayos X, MEB y MET, además de las variables usadas en la experimentación (temperatura $\left({ }^{\circ} \mathrm{C}\right)$, presión $(\mathrm{atm})$ y flujo de gas inyectado 
R. Muñoz-Arroyo, J.C. Escobedo-Bocardo, H.M. Hernández-García, D.A. Cortés-Hernández, M. TeRrones-Maldonado, A. RodRíguez-Pulido y J.L. HeRnándeZ-PIÑERo

Tabla II. Eficiencia en la remoción de magnesio y mermas de aleación para cada uno de los experimentos efectuados

Table II. Efficiency of magnesio removal and metal losses from the alloy for each experiment

\begin{tabular}{lccccccc}
\hline \multicolumn{1}{c}{ MUESTRA } & $\begin{array}{c}\text { Peso } \\
\text { inicial } \\
(\mathbf{k g})\end{array}$ & $\begin{array}{c}\text { Peso } \\
\text { lingote } \\
\mathbf{( k g )}\end{array}$ & $\begin{array}{c}\text { Peso } \\
\text { probetas } \\
(\mathbf{k g})\end{array}$ & $\begin{array}{c}\text { Peso } \\
\text { escoria } \\
(\mathbf{k g})\end{array}$ & $\begin{array}{c}\text { Contenido } \\
\text { Final de } \\
\text { Mg(\%e.p.) }\end{array}$ & $\begin{array}{c}\text { Eficiencia } \\
(\%)\end{array}$ & Merma (\%) \\
\hline 50Sílice:50Zeolita & 8,000 & 6,150 & 1,350 & 1,010 & 0,0541 & 94,59 & 6,2500 \\
34Sílice:66Zeolita & 8,100 & 6,300 & 1,165 & 1,088 & 0,0066 & 99,34 & 7,8395 \\
Zeolita & 8,000 & 5,600 & 1,080 & 1,281 & 0,0280 & 97,20 & 16,500 \\
Sílice & 8,000 & 5,800 & 0,857 & 1,240 & 0,1060 & 89,40 & 16,7875 \\
\hline
\end{tabular}

Tabla III. Compuestos encontrados en la superficie de los cubos de zeolita identificados por difracción de rayos $X$

Table III. Compounds on the zeolite cubes surface detected by XRD

\begin{tabular}{lccccc}
\hline \multicolumn{1}{c}{ Compuestos o elementos } & $\mathbf{1 0}(\mathrm{min})$ & $\mathbf{4 0}(\mathrm{min})$ & $\mathbf{6 0}(\mathbf{m i n})$ & $\mathbf{8 0}(\mathbf{m i n})$ & $\mathbf{9 0}$ (min) \\
\hline $\mathrm{SiO}_{2}$ (cuarzo) & $\mathrm{X}$ & $\mathrm{X}$ & $\mathrm{X}$ & $\mathrm{X}$ & $\mathrm{X}$ \\
$\mathrm{Al}$ & $\mathrm{X}$ & $\mathrm{X}$ & $\mathrm{X}$ & $\mathrm{X}$ & $\mathrm{X}$ \\
$\mathrm{SiO}_{2 \text { (cristobalita) }}$ & $\mathrm{X}$ & & & & \\
$\mathrm{MgO}_{\text {(periclasa) }}$ & $\mathrm{X}$ & $\mathrm{X}$ & $\mathrm{X}$ & $\mathrm{X}$ & $\mathrm{X}$ \\
$\mathrm{CaAl}_{2} \mathrm{Si}_{2} \mathrm{O}_{8 \text { (anortita) }}$ & & & $\mathrm{X}$ & $\mathrm{X}$ & $\mathrm{X}$ \\
$\mathrm{Si}_{\mathrm{CaAl}} \mathrm{Si}_{7} \mathrm{O}_{18} \cdot 6 \mathrm{H}_{2} \mathrm{O}_{\text {(heulandita) }}$ & & & $\mathrm{X}$ & $\mathrm{X}$ & $\mathrm{X}$ \\
$\mathrm{KAISi}_{3} \mathrm{O}_{8 \text { (sanidina) }}$ & & & & & $\mathrm{X}$ \\
\hline
\end{tabular}

$(\mathrm{Ar}))$, se computaron una serie de reacciones químicas en los módulos de equilibrio y reacción del paquete FactSage, a partir de la composición química de los minerales. Las reacciones se establecieron en base a las magnitudes de las energías libres de formación en el equilibrio.

La elevada eficiencia de la zeolita como agente eliminador de magnesio se debe a dos de sus características: la elevada superficie de reacción que presenta este mineral y la presencia de agua molecular atrapada en su estructura. De lo anterior, se estableció una fórmula general que representa la naturaleza hidratada del mineral ${ }^{[8}$ y 9$]$ :

$$
\left(\mathrm{Na}_{2}, \mathrm{~K}_{2}, \mathrm{Ca}, \mathrm{Cl}\right)[(\mathrm{Al}, \mathrm{Si}, \mathrm{Mg}) \mathrm{On}]\left(\mathrm{H}_{2} \mathrm{O}\right)_{n}
$$

Con base en los productos de reacción encontrados y en concordancia con los cálculos realizados en el módulo de reacción del software FactSage, se sugiere que la eliminación del magnesio ocurre de la siguiente manera:

$$
\begin{aligned}
& \mathrm{CaAl}_{2} \mathrm{Si}_{7} \mathrm{O}_{18} \cdot 6 \mathrm{H}_{2} \mathrm{O}_{\text {(heulandita) }}+2 \mathrm{Mg}_{(l)}+2 \mathrm{Al}_{(l)}+ \\
& \mathrm{Ar}_{(g)} \rightarrow \mathrm{CaAl}_{2} \mathrm{Si}_{2} \mathrm{O}_{8(\text { anortita) }}+\mathrm{MgO}_{(\text {periclasa) }}+ \\
& \mathrm{MgAl}_{2} \mathrm{O}_{4(\text { espinela) }}+5 \mathrm{SiO}_{2}+6 \mathrm{H}_{2(\mathrm{~g})}+0.5 \mathrm{O}_{2(g)}+ \\
& \operatorname{Ar}_{(g)} \Delta \mathrm{G}^{\circ}{ }_{750^{\circ} \mathrm{C}}=-2081,992 \mathrm{kj}
\end{aligned}
$$

Se sugiere que durante la deshidratación del mineral dentro del aluminio líquido $\left(\mathrm{Al}_{(1)}\right)$ se forma una capa de vapor alrededor del mineral que actúa como agente oxidante, dando lugar a la formación de óxidos tales como $\mathrm{MgO}, \mathrm{Mg}_{2} \mathrm{SiO}_{4}, \mathrm{MgAl}_{2} \mathrm{O}_{4}$, $\mathrm{Al}_{2} \mathrm{O}_{3}$, etc., que se forman en la superficie del mineral y parte de estos se van a la escoria (Fig. 6).

Ocurren en este sitio las siguientes reacciones: 


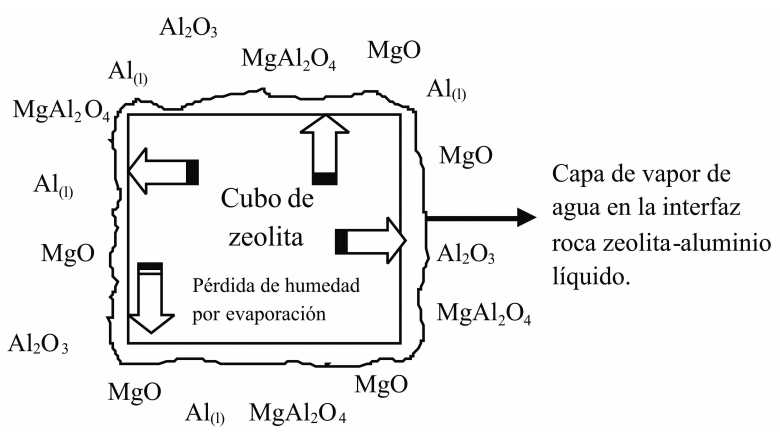

Figura 6. Esquema de la capa de vapor de agua en la interfaz zeolita-aluminio líquido.

Figure 6. Diagram showing the water steam layer at the interface zeolite-aluminum liquid.

$$
\begin{aligned}
& 2\left[\mathrm{~K}, \mathrm{Cl}\left(\mathrm{AlSi}_{3} \mathrm{O}_{8(\text { sanidina) }}\right)\right]+4 \mathrm{Mg}_{(\mathrm{l})}+2 \mathrm{Al}{ }_{(I)} \rightarrow \\
& \mathrm{Mg}_{2} \mathrm{SiO}_{4(\text { forsterita) }}+\mathrm{MgAl}_{2} \mathrm{O}_{4(\text { espinela) }}+\mathrm{MgO}_{(\text {periclasa) }}(4) \\
& +\mathrm{Al}_{2} \mathrm{O}_{3 \text { (corundo) }}+2 \mathrm{KCl}_{(\text {silvita) }}+2 \mathrm{SiO}_{2}+3 \mathrm{Si}_{(\mathrm{s})} \\
& \Delta \mathrm{G}^{\circ}{ }_{750^{\circ} \mathrm{C}}=-1266,419 \mathrm{~kJ}
\end{aligned}
$$

Estas reacciones sugieren la disminución del contenido de magnesio, que es debido, principalmente, al efecto del $\mathrm{H}_{2} \mathrm{O}$. Conforme se incrementa el tiempo de inmersión de los minerales en el aluminio líquido y ocurre la deshidratación del mineral, interviene el $\mathrm{SiO}_{2}$ de acuerdo a las siguientes reacciones:

$$
\begin{aligned}
& 4.5 \mathrm{SiO}_{2 \text { (cuarzo) }}+4 \mathrm{Mg}(\mathrm{l})+2 \mathrm{Al}(\mathrm{l})+\mathrm{Ar}(\mathrm{g}) \rightarrow \\
& \mathrm{Mg}_{2} \mathrm{SiO}_{4 \text { (forsterita) }}+\mathrm{MgO}_{\text {(periclasa) }}+\mathrm{MgAl}_{2} \mathrm{O}_{4 \text { (espinela) }} \\
& +3.5 \mathrm{Si}+\mathrm{Ar}_{(\mathrm{g})} \Delta \mathrm{G}^{\circ}{ }_{750^{\circ} \mathrm{C}}=-850.340 \mathrm{~kJ} \\
& \mathrm{SiO}_{2 \text { (amorfo) }}+\mathrm{MgAl}_{2} \mathrm{O}_{4(\text { espinela) }}+2 \mathrm{Al}_{(\mathrm{l})}+0.5 \mathrm{O}_{2(\mathrm{~g})} \\
& +\mathrm{Ar}_{(g)} \rightarrow \mathrm{MgO}_{\text {(periclasa) }}+2 \mathrm{Al}_{2} \mathrm{O}_{3}+\mathrm{Si}_{(\mathrm{s})}+\mathrm{Ar}_{(\mathrm{g})} \\
& \Delta \mathrm{G}^{\circ} 750^{\circ} \mathrm{C}=-603.931
\end{aligned}
$$

\subsection{Microscopía electrónica transmisión}

La figura 7 a), b), c) y d) muestra una imagen de microscopía electrónica de transmisión de una partícula de la mezcla de minerales sílice: zeolita, 34:66 \% e.p., la cual estuvo sumergida en el baño líquido de aluminio por $10 \mathrm{~min}$ a $750^{\circ} \mathrm{C}$. Se observan fracturas en relieves del mineral y productos de reacción de morfología áspera sobre la superficie de la partícula (Fig. 7 a) y b)).

La variación de los contenidos de los elementos en \%e.p. evidencia el inicio de la formación de

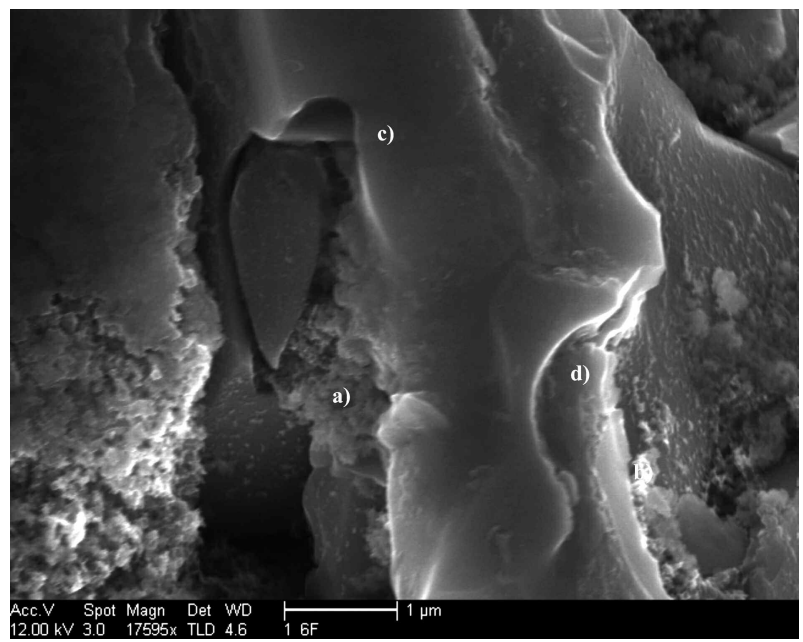

Figura 7. Imagen de microscopía electrónica de transmisión de una partícula de mineral sumergida por 10 minutos en la aleación de aluminio líquida: a) y b) Productos de reacción; c) y d) Superficie del mineral.

Figure 7. TEM image of a submerged particle after 10 min in the aluminum liquid alloy. a) and b) Reaction products and c) and d) Mineral surface.

partículas menores a $1 \mu \mathrm{m}$, de productos de estequiometria compleja sobre áreas diversas del mineral (Tabla IV). Evidentemente, se aprecia en la tabla IV que, en estas partículas, se incrementan los contenidos de magnesio y aluminio con una disminución de oxígeno y silicio, a medida que nos alejamos del centro de la partícula (de la zona a) a la zona d)).

Se observan, en la tabla IV, otras especies químicas (elementos metálicos) como cobre, níquel y hierro que interaccionan por intercambio iónico con el mineral, formando parte de los productos de reacción. Lo anterior, evidencia que el tiempo de permanencia de $10 \mathrm{~min}$ de las partículas en el baño líquido de aluminio no fue suficiente para lograr una reacción completa y, así, formar capas óxidos de alta estabilidad térmica.

La figura 8 muestra una imagen de microscopía electrónica de transmisión de una partícula de la mezcla de minerales sílice:zeolita 34:66 \% e.p. sumergida en el baño de aluminio líquido, por $40 \mathrm{~min}$ a $750^{\circ} \mathrm{C}$. Se observa que se inicia la formación de capas sobre el mineral. En el análisis puntual en el sentido de la trayectoria de la flecha, se aprecian, en cada capa, diferencias en los contenidos de los elementos (Tabla V).

En la Tabla V se aprecia el incremento de los contenidos de magnesio y oxígeno de la muestra de la figura 8. En contraste, los contenidos de silicio 
R. Muñoz-Arroyo, J.C. Escobedo-Bocardo, H.M. Hernández-García, D.A. Cortés-Hernández,

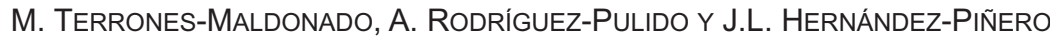

Tabla IV. Contenidos de los elementos presentes en las zonas analizadas de partículas sumergidas en aluminio líquido por $10 \mathrm{~min}$ a $750{ }^{\circ} \mathrm{C}$

Table IV. Elements content in the analyzed zones on particles submerged in aluminum liquid for $10 \mathrm{~min}$ at $750^{\circ} \mathrm{C}$

\begin{tabular}{|c|c|c|c|c|c|c|c|c|}
\hline Zona & $\begin{array}{c}\text { Al } \\
(\% \text { e.p. })\end{array}$ & $\begin{array}{c}\mathrm{Si} \\
(\% \text { e.p. })\end{array}$ & $\begin{array}{c}0 \\
(\% \text { e.p. })\end{array}$ & $\begin{array}{c}\mathrm{Mg} \\
\text { (\%e.p.) }\end{array}$ & $\begin{array}{c}\mathrm{Cu} \\
(\% \text { e.p. })\end{array}$ & $\begin{array}{c}\mathrm{Ni} \\
(\% \text { e.p. })\end{array}$ & $\begin{array}{c}\mathrm{Fe} \\
\text { (\%e.p.) }\end{array}$ & Compuesto probable \\
\hline A & 34,17 & 60,9887 & 2,3741 & 0,8797 & 1,2304 & 0,0659 & 0,2830 & $\left.{ }_{35} \mathrm{Mg}_{0,1} \mathrm{Fe}_{0,014} \mathrm{Ni}_{0,0031}\right)$ \\
\hline B & 30,9034 & 60,3146 & 3,0923 & 0,5655 & 1,0735 & 3,8028 & 0,2479 & $\left.{ }_{2} \mathrm{Mg}_{0,065} \mathrm{Fe}_{0,0124} \mathrm{Ni}_{0,181}\right)$ \\
\hline C & 18,9709 & 69,1149 & 7,4995 & 0,2373 & 1,2163 & 0,7796 & 2,1815 & $\mathrm{Al}_{3,6} \mathrm{Si}_{12,6} \mathrm{O}_{2,4}\left(\mathrm{Cu}_{0,098} \mathrm{Mg}_{0,05} \mathrm{Fe}_{0,2} \mathrm{Ni}_{0,068}\right)$ \\
\hline D & 17,9202 & 70,3502 & 6,9830 & 0,2214 & 1,0853 & 1,0025 & 2,4375 & $\mathrm{Al}_{1,7} \mathrm{Si}_{6,6} \mathrm{O}_{1,15}\left(\mathrm{Cu}_{0,045} \mathrm{Mg}_{0,024} \mathrm{Fe}_{0,115} \mathrm{Ni}_{0,045}\right)$ \\
\hline
\end{tabular}

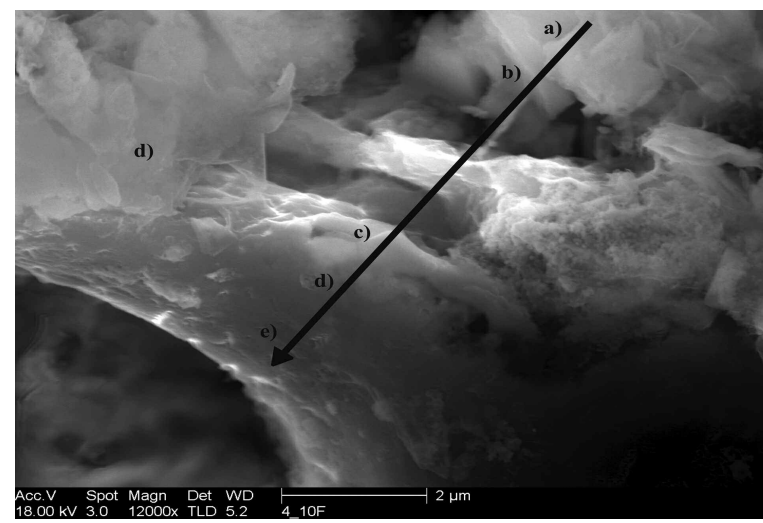

Figura 8. Imagen de microscopia electrónica de barrido (modo STEM) de alta resolución de una partícula sumergida por $40 \mathrm{~min}$ a $750^{\circ} \mathrm{C}$ en aluminio líquido: a), b), c), d) y e) Partículas de compuestos complejos formando capas por intercambios iónicos entre el mineral y especies disueltas en baño líquido de aluminio.

Figure 8. TEM image of a submerged particle for $40 \mathrm{~min}$ at $750^{\circ} \mathrm{C}$ in the aluminum liquid. a), b), c), d) and e) Complex compounds forming layers by ionic exchanges between mineral and species in the aluminum liquid bath. disminuyen (conforme se avanza de a) en dirección a e)). Se observa que los contenidos de aluminio varían debido a la presencia de la aleación base aluminio.

\section{CONCLUSIONES}

La elevada área superficial, la facilidad para el intercambio iónico y la presencia de agua en la zeolita, aunado a la topografía de las maclas de Carlsbad, son factores que hacen de ella un efectivo eliminador de magnesio de las aleaciones de aluminio líquidas. Sin embargo, si la cantidad de agua no se controla la oxidación excesiva del baño puede conducir a mermas tan elevadas que hagan inviable el proceso. Por tanto, es mejor emplear mezclas de zeolita más sílice, resultando en este estudio la mejor mezcla la de 34 $\%$ e.p. sílice $+66 \%$ e.p. zeolita, con un contenido final de magnesio de 0,0066\%e.p.

El planteamiento de las reacciones químicas desarrollado en el paquete FactSage es consistente con los productos de reacción reportados por difracción de rayos X, MEB, STEM y MET. Los cálculos termodinámicos realizado en el paquete FactSage

Tabla V. Contenidos de los elementos presentes en las zonas analizadas de partículas inmersas en aluminio líquido por $40 \mathrm{~min}$ a $750^{\circ} \mathrm{C}$ y sus probables compuestos

Table V. Elements content on selected zones of particles submerged in aluminum liquid for $40 \mathrm{~min}$ at $750^{\circ} \mathrm{C}$ and their probable compounds

\begin{tabular}{ccccccc}
\hline Zona & $\begin{array}{c}\text { Al } \\
\text { (\%e.p.) }\end{array}$ & $\begin{array}{c}\text { Si } \\
\text { (\%e.p.) }\end{array}$ & $\begin{array}{c}\text { O } \\
\text { (\%e.p.) }\end{array}$ & $\begin{array}{c}\text { Mg } \\
\text { (\%e.p.) }\end{array}$ & $\begin{array}{c}\text { Cu } \\
\text { (\%e.p.) }\end{array}$ & Compuesto probable \\
\hline a & 29,2566 & 50,1876 & 14,4339 & 4,7438 & 1,3781 & $\mathrm{Mg}_{1,8} \mathrm{Al}_{10} \mathrm{Si}_{16,48} \mathrm{O}_{8,32}\left(\mathrm{Cu}_{0,2}\right)$ \\
$\mathrm{b}$ & 34,4426 & 46,3173 & 14,0737 & 2,4821 & 2,6843 & $\mathrm{MgAM}_{11} \mathrm{Si}_{14,211} \mathrm{O}_{7,58}\left(\mathrm{Cu}_{0,364}\right)$ \\
c & 36,4189 & 27,3703 & 22,1354 & 11,4165 & 2,6589 & $\mathrm{Mg}_{3,48} \mathrm{Al}_{10} \mathrm{Si}_{7,22} \mathrm{O}_{10,25}\left(\mathrm{Cu}_{0,31}\right)$ \\
d & 25,3525 & 29,5748 & 27,6304 & 14,7657 & 2,6766 & $\mathrm{Mg}_{3,75} \mathrm{Al}_{5,8} \mathrm{Si}_{6,5} \mathrm{O}_{10,66}\left(\mathrm{Cu}_{0,21}\right)$ \\
e & 19,7918 & 28,3271 & 32,2741 & 17,1599 & 2,4472 & $\mathrm{Mg}_{3,85} \mathrm{Al}_{4} \mathrm{Si}_{5,5} \mathrm{O}_{11}\left(\mathrm{Cu}_{0,21}\right)$ \\
\hline
\end{tabular}


corroboraron en base a las magnitudes de las energías libres de formación estándar, el efecto del $\mathrm{SiO}_{2}$ de la heulandita y sanidina para la formación de óxidos de alta estabilidad térmica como son: $\mathrm{MgAl}_{2} \mathrm{O}_{4}$ (espinela), $\mathrm{MgO}_{\text {(periclasa) }}, \mathrm{Mg}_{2} \mathrm{SiO}_{4}$ (forsterita), $\mathrm{Al}_{2} \mathrm{O}_{3}$ (alúmina), etc.

\section{REFERENCIAS}

[1] V.D. Neff V. y P.B. Cochran, Light Metals, Light Metals Committee at the $122^{\text {nd }}$ TMS Annual Meeting TMS, EE. UU., 1993, pp. 1.053-1.060.

[2] B.L. Tiwari, B.J. Howie y R.M. Johnson, Proc. The Ninetieth Annual Meeting AFS Transactions, Illinois, EE. UU., 1986, William O’Neill (Ed.), Illinois, EE. UU., 1986, pp. 385-390.

[3] T.R. George, T.R. Koros y R.G. Petrushka, Inter. Symp. Injection Process Metall., Vol. 1, Pennsylvania, EE. UU., 1991, T. Lehner, J. Koros y V. Ramachandran (Eds.), T. M. S., Pennsylvania, EE. UU., 1991, pp. 81-98.
[4] O.J. Ilegbusi, J. Szekely, Inter. Symp. Injection Process Metall., Vol. 2, Pennsylvania, EE. UU., 1991, T. Lehner, J. Koros y V. Ramachandran (Eds.), T. M. S., Pennsylvania, EE. UU., 1991, pp. 1-34.

[5] R. Muñoz, J.C. Escobedo, H.M. Hernández, D.A. Hernández, J.M. Almanza, J. Donizak y Z. Kolenda, Arch. Metall. Mater. 53 (2008) $965-$ 968.

[6] J.C. Escobedo, J.F. Hernández, S. Escobedo, A. Flores y D.A. Hernández, Rev. Metal. Madrid 39 (2003) 172-182.

[7] J.C. Escobedo, A. Flores, D.A. Hernández y J.J. Velázquez, Light Metals, Light Metals Committee at the $132^{\text {nd }}$ TMS Annual Meeting TMS, EE. UU., 2003, pp. 885-891.

[8] R. Muñoz, Tesis Doctoral, Centro de Investigación y de Estudios Avanzados del IPN, Unidad Saltillo, México, 2009.

[9] H.R. Wenk y A. Bulakh, Minerals, Ed. Cambridge, Tercera edición, Londres, Inglaterra, 2008, pp. 3-11, 32-89, 313-343 y 425-508. 